Pacific

Journal of

Mathematics

UNIVERSAL DEFORMATION FORMULAE, SYMPLECTIC LIE GROUPS AND SYMMETRIC SPACES

Pierre Bieliavsky, Philippe Bonneau and Yoshiaki Maeda 


\title{
UNIVERSAL DEFORMATION FORMULAE, SYMPLECTIC LIE GROUPS AND SYMMETRIC SPACES
}

\author{
Pierre Bieliavsky, Philippe Bonneau And Yoshiaki Maeda
}

\begin{abstract}
We define a class of symplectic Lie groups associated with solvable symmetric spaces. We give a universal strict deformation formula for every proper action of such a group on a smooth manifold. We define a functional space where performing an asymptotic expansion of the nonformal deformed product in powers of the deformation parameter yields an associative formal star product on the symplectic Lie group at hand. The cochains of the star product are explicitly given (without recursion) in the two-dimensional case of the affine group $a x+b$. The latter differs from the Giaquinto-Zhang construction, as shown by analyzing the invariance groups. In a Hopf algebra context, the above formal star product is shown to be a smash product and a compatible coproduct is constructed.
\end{abstract}

\section{Introduction}

The concept of universal deformation for abelian Lie group actions was introduced by Rieffel [1993] in the operator algebraic context. Later, the notion of a universal deformation formula (UDF) within a Hopf algebraic context was defined by Giaquinto and Zhang [1998] at the formal level. Here we study a similar notion within the framework of (nonabelian) solvable Lie group actions. Our construction of UDFs relies on both formal and nonformal aspects. The Lie groups considered here are symplectic semidirect products of abelian Lie groups, natural generalizations of the two-dimensional affine group $a x+b$. On each such group $\mathbb{S}$, we define a function space $\mathbb{A} \subset C^{\infty}(\mathbb{S})$ invariant under the left regular representation and endowed with a one-parameter family of products $\left\{\star_{\theta}\right\}_{\theta \in \mathbb{R}}$ in such a way that each pair $\left(\mathbb{A}, \star_{\theta}\right)$ is an associative (topological) algebra which the group $\mathbb{S}$ naturally acts on by automorphisms. As observed in [Bieliavsky 2002; Bieliavsky et al. 2003], such a data provides a UDF in the following way. Let $M$ denote

MSC2000: primary 54C40, 14E20; secondary 46E25, $20 \mathrm{C} 20$.

Keywords: universal deformation formula, symplectic Lie group, symmetric space, Lie group actions.

Maeda is partially supported by the 21th Century COE Program "Integrative Mathematical Sciences, Progress in Mathematics Motivated by Natural and Social Phenomena", and by Grant-in-Aid for Scientific Research \#18204006, Ministry of Education, Science and Culture, Japan. 
a smooth manifold which the group $\mathbb{S}$ acts on by diffeomorphisms. Denote by $\alpha: \mathbb{S} \times C^{\infty}(M) \rightarrow C^{\infty}(M):(s, u) \mapsto \alpha_{s}(u)$ the induced action at the level of functions and set $\alpha^{x}(u)(s):=\alpha_{s}(u)(x)$ for $u \in C^{\infty}(M), x \in M$ and $s \in \mathbb{S}$. Then, the function space $\mathbb{B}:=\left\{u \in C^{\infty}(M)\right.$ such that $\alpha^{x}(u) \in \mathbb{A}$ for all $\left.x \in M\right\}$ is naturally endowed with a one-parameter family of associative products $\left\{\star_{\theta}^{M}\right\}$ defined by the formula $u \star_{\theta}^{M} v(x):=\left(\alpha^{x}(u) \star_{\theta} \alpha^{x}(v)\right)(e)$ ( $e$ denotes the identity element in $\mathbb{S}$ ). The problem is of course to show that the space $\mathbb{B}$ is sufficiently rich, in the sense that it contains at least the smooth compactly supported functions on $M$. This is the case for the class of groups $\mathbb{S}$ considered here provided the action of $\mathbb{S}$ on $M$ is proper. Next, comes the question of defining an appropriate functional framework allowing to pass from our nonformal setting to the formal framework of star products [Bayen et al. 1978a; 1978b]. The above-mentioned nonformal universal deformation formulae are of the oscillatory - or WKB - type. This means that the product $a \star_{\theta} b$ is defined by an integral expression $a \star_{\theta} b=\int_{\mathbb{S} \times \mathbb{S}} K_{\theta} a \otimes b$ where the kernel $K_{\theta}$ has the oscillatory form $K_{\theta}=\theta^{-m} \mathbf{a}_{\theta} e^{(i / \theta) S}$, where $\mathbf{a}_{\theta}$ and $S$ belong to $C^{\infty}(\mathbb{S} \times \mathbb{S} \times \mathbb{S}, \mathbb{R})$. In particular, for $a$ and $b$ smooth compactly supported, one may perform a stationary phase expansion of $a \star_{\theta} b$ in powers of $\theta$ yielding a formal product on $C^{\infty}(\mathbb{S}) \llbracket \theta \rrbracket$. The property of associativity of this formal product depends on functional properties of the nonformal algebras $\left(\mathbb{A}, \star_{\theta}\right)$.

In Section 2, we prove that every exact symplectic semidirect product $\mathbb{S}$ of two abelian groups always acts strictly transitively on an elementary solvable symplectic symmetric space in the sense of [Bieliavsky 2002]. Conversely, we show that when complex every such space gives rise to an exact symplectic semidirect product of two abelian groups. This allows to identify in a $\mathbb{S}$-equivariant manner the manifolds underlying the group $\mathbb{S}$ and the corresponding symmetric space.

In Section 3, we observe that Section 2 together with the construction in [Bieliavsky 2002] of nonformal quantizations on elementary solvable symplectic symmetric spaces yield on every such semidirect product $\mathbb{S}$ a left-invariant nonformal deformation quantization. As an immediate consequence, the latter gives rise to strict deformation quantizations for the proper actions of $\mathbb{S}$ on smooth manifolds.

In Section 4, restricting to the case $\operatorname{dim} S=2$, we associate to our nonformal deformation an associative left-invariant formal star product on $C^{\infty}(\mathbb{S}) \llbracket \theta \rrbracket$ for which we give the cochains totally explicitly (without any recursion). This is essentially done by defining a $\theta$-independent functional space (denoted hereafter $\mathscr{E}$ ) closed under our one-parameter family of nonformal deformations $\left\{\star_{\theta}\right\}$ and by studying the smoothness (in a suitable sense) of our algebras $\left\{\left(\mathscr{E}, \star_{\theta}\right)\right\}_{\theta}$. For nonzero values of $\theta$, the space $\mathscr{E}$ plays an analogous role as the Schwartz space does in the case of Weyl's quantization. As it clearly appears, the restriction to the dimension two is inessential. 
In Section 5, we show that the above mentioned formal star product can be seen as a smash product of $C^{\infty}(\mathbb{R}) \llbracket \theta \rrbracket$ and the Hopf algebra of polynomials on $\mathbb{R}$. From the latter, we deduce a compatible formal coproduct and a Hopf structure on $C^{\infty}(\mathbb{S}) \llbracket \theta \rrbracket$.

\section{Symplectic Lie algebras associated to a class of symmetric spaces}

Definition 2.1. Following the terminology of [Lichnerowicz and Medina 1988], a symplectic Lie algebra is a pair $(\mathfrak{s}, \omega)$ where $\mathfrak{s}$ is a Lie algebra and $\omega \in \wedge^{2}\left(\mathfrak{s}^{\star}\right)$ is a nondegenerate Chevalley two-cocycle with respect to the trivial representation of $\mathfrak{s}$.

In this section, we associate symplectic Lie algebras to a class of (infinitesimal) symplectic symmetric spaces.

Definition 2.2 [Bieliavsky et al. 1995; Bieliavsky 1995]. Let $(\mathfrak{g}, \sigma)$ be an involutive algebra, meaning that $\mathfrak{g}$ is a finite dimensional real Lie algebra and $\sigma$ is an involutive automorphism of $\mathfrak{g}$. Let $\Omega$ be a skewsymmetric bilinear form on $\mathfrak{g}$. Then the triple $(\mathfrak{g}, \sigma, \Omega)$ is called a symplectic triple if the following properties are satisfied.

(i) Let $\mathfrak{g}=\mathfrak{k} \oplus \mathfrak{p}$ where $\mathfrak{k}$ (resp. $\mathfrak{p}$ ) is the +1 (resp. -1) eigenspace of $\sigma$. Then $[\mathfrak{p}, \mathfrak{p}]=\mathfrak{k}$ and the representation of $\mathfrak{k}$ on $\mathfrak{p}$, given by the adjoint action, is faithful.

(ii) $\Omega$ is a Chevalley 2-cocycle for the trivial representation of $\mathfrak{g}$ on $\mathbb{R}$ such that for any $X$ in $\mathfrak{k}, i(X) \Omega=0$. Moreover, the restriction of $\Omega$ to $\mathfrak{p} \times \mathfrak{p}$ is nondegenerate.

Two such triples $\left(\mathfrak{g}_{i}, \sigma_{i}, \Omega_{i}\right)(i=1,2)$ are isomorphic if there exists a Lie algebra isomorphism $\psi: \mathfrak{g}_{1} \rightarrow \mathfrak{g}_{2}$ such that $\psi \circ \sigma_{1}=\sigma_{2} \circ \psi$ and $\psi^{*} \Omega_{2}=\Omega_{1}$.

Such a triple is called indecomposable if it cannot be expressed as a direct sum of symplectic triples.

Definition 2.3. A symplectic triple $t=(\mathfrak{g}, \sigma, \Omega)$ is called holonomy isotropic, or $\mathrm{HI}$, if $[\mathfrak{k}, \mathfrak{p}]$ is an isotropic subspace of $(\mathfrak{p}, \Omega)$.

Proposition 2.1 [Bieliavsky 1998]. A symplectic triple $t=(\mathfrak{g}, \sigma, \Omega)$ is holonomy isotropic if and only if $[\mathfrak{g}, \mathfrak{g}]$ is abelian.

Definition 2.4. Let $t=(\mathfrak{g}, \sigma, \Omega)$ be $\mathrm{HI}$ and consider the extension sequence

$$
0 \rightarrow[\mathfrak{g}, \mathfrak{g}] \rightarrow \mathfrak{g} \rightarrow \mathfrak{a}:=\mathfrak{g} /[\mathfrak{g}, \mathfrak{g}] \rightarrow 0 .
$$

The HI triple $t$ is called split if this extension is split.

Lemma 2.2. Let $t=(\mathfrak{g}, \sigma, \Omega)$ be HI split. Set $\mathfrak{b}=[\mathfrak{g}, \mathfrak{g}]$ and denote by $\rho: \mathfrak{a} \rightarrow$ $\operatorname{End}(\mathfrak{b})$ the splitting homomorphism. Then, realizing $\mathfrak{g}$ as the semidirect product $\mathfrak{g}=\mathfrak{b} \times{ }_{\rho} \mathfrak{a}$, one can assume that $\mathfrak{a}$ is stable under $\sigma$. 
Proof. For $a \in \mathfrak{a} \subset \mathfrak{g}$, write $a=a_{\mathfrak{k}}+a_{\mathfrak{p}}$ according to the decomposition with respect to $\sigma$. Then for all $a, a^{\prime} \in \mathfrak{a}$, one has $0=\left[a, a^{\prime}\right]=\left[a_{\mathfrak{p}}, a_{\mathfrak{p}}^{\prime}\right]+b \quad b \in[\mathfrak{k}, \mathfrak{p}]$ since $\mathfrak{k}$ is abelian. This yields $\left[a_{\mathfrak{p}}, a_{\mathfrak{p}}^{\prime}\right]=0$.

Therefore, for $\operatorname{pr}_{\mathfrak{p}}: \mathfrak{g} \rightarrow \mathfrak{p}$ the projection parallel to $\mathfrak{k}$, the $\mathfrak{p}$-component $\operatorname{pr}_{\mathfrak{p}}(\mathfrak{a})$ is an abelian subalgebra of $\mathfrak{g}$ supplementary to $\mathfrak{b}$. A dimension count then yields the lemma.

Lemma 2.3. Assume that $t=(\mathfrak{g}, \sigma, \Omega)$ is HI split, indecomposable and nonflat. Set $0 \rightarrow \mathfrak{b} \rightarrow \mathfrak{g} \rightarrow \mathfrak{a} \rightarrow 0$ as in Lemma 2.2. Then $\mathfrak{a}$ and $\mathfrak{l}=[\mathfrak{k}, \mathfrak{p}]$ are in duality. In particular, there exists a $\mathfrak{k}$-invariant symplectic structure on $\mathfrak{p}$ for which $\mathfrak{a}$ is Lagrangian.

Proof. Set $V:=\mathfrak{l}^{\perp} \cap \mathfrak{a}$ and choose a subspace $W$ of $\mathfrak{a}$ in duality with $\mathfrak{l}$. Counting dimensions yields $\mathfrak{a}=W \oplus V$. Moreover, in the decomposition $\mathfrak{p}=\mathfrak{l} \oplus W \oplus V$, the matrix of $\Omega$ is of the form

$$
[\Omega]=\left(\begin{array}{rrr}
0 & I & 0 \\
-I & 0 & B \\
0 & -B^{\prime} & A
\end{array}\right) .
$$

Since $\operatorname{det}[\Omega] \neq 0$, one gets $\operatorname{det}\left(\begin{array}{rr}-I & B \\ 0 & A\end{array}\right) \neq 0$; hence $\operatorname{det} A \neq 0$ and $V$ is symplectic. Now, $\Omega([\mathfrak{k}, V], \mathfrak{p})=\Omega(V, \mathfrak{l})=0$, hence $[\mathfrak{k}, V]=0$. Also $[V, \mathfrak{l}]=[V,[\mathfrak{k}, \mathfrak{p}]]=0$ by Jacobi. Thus $V$ is central, and therefore trivial by indecomposability.

We now assume that $\left(\mathfrak{g}^{1}, \sigma^{1}\right)$ is the involutive Lie algebra underlying a split HI symplectic triple which is indecomposable and nonflat. We fix $\Omega^{1}$ such that the HI symplectic triple $t^{1}=\left(\mathfrak{g}^{1}, \sigma^{1}, \Omega^{1}\right)$ with $0 \rightarrow \mathfrak{b}^{1} \rightarrow \mathfrak{g}^{1} \rightarrow \mathfrak{a}^{1} \rightarrow 0$ has $\mathfrak{a}^{1}$ and $\mathfrak{l}^{1}=\left[\mathfrak{k}^{1}, \mathfrak{p}^{1}\right]$ dual Lagrangian subspaces. We then consider the associated exact triple [Bieliavsky 1998], which we denote by $t=(\mathfrak{g}, \sigma, \Omega)$ (if $t^{1}$ is already exact we set $\left.t=t^{1}\right)$. Since $\mathfrak{a}^{1}$ is isotropic, the triple $t$ is elementary solvable with

$$
0 \rightarrow \mathfrak{b}:=[\mathfrak{g}, \mathfrak{g}] \rightarrow \mathfrak{g} \rightarrow \mathfrak{a}:=\mathfrak{a}^{1} \rightarrow 0 .
$$

We now follow a procedure as in [Bieliavsky 2002]. The map $\rho: \mathfrak{a} \rightarrow \operatorname{End}(\mathfrak{b})$ is injective (because $\Omega$ is nondegenerate), so we may identify $\mathfrak{a}$ with its image $: \mathfrak{a}=$ $\rho(\mathfrak{a})$. Let $\Sigma: \operatorname{End}(\mathfrak{b}) \rightarrow \operatorname{End}(\mathfrak{b})$ be the automorphism induced by the conjugation with respect to the involution $\left.\sigma\right|_{\mathfrak{b}} \in G L(\mathfrak{b})$, i.e. $\Sigma=A d\left(\left.\sigma\right|_{\mathfrak{b}}\right)$. The automorphism $\Sigma$ is involutive and preserves the canonical Levi decomposition $\operatorname{End}(\mathfrak{b})=\mathscr{L} \oplus \operatorname{sl}(\mathfrak{b})$, where $\mathscr{L}$ denotes the center of $\operatorname{End}(\mathfrak{b})$. Writing the element $a=\rho(a) \in \mathfrak{a}$ as $a=a_{Z}+a_{0}$ with respect to this decomposition, one has $\Sigma(a)=a_{Z}+\Sigma\left(a_{0}\right)=$ $-a=-a_{Z}-a_{0}$, because the endomorphisms $a$ and $\left.\sigma\right|_{\mathfrak{b}}$ anticommute. Hence $\Sigma\left(a_{0}\right)=-2 a_{Z}-a_{0}$ and therefore $a_{Z}=0$. So, $\mathfrak{a}$ actually lies in the semisimple part $\operatorname{sl}(\mathfrak{b})$. For any $x \in \operatorname{sl}(\mathfrak{b})$, we denote by $x=x^{S}+x^{N}, \quad x^{S}, x^{N} \in \operatorname{sl}(\mathfrak{b})$, its abstract Jordan-Chevalley decomposition. Observe that, for $s l(\mathfrak{b})=s l_{+} \oplus s l_{-}$, the 
decomposition in $( \pm 1)$ - $\Sigma$ - eigenspaces, one has $\mathfrak{a} \subset s l_{-}$. Also, $\mathfrak{a}_{N}:=\left\{a^{N}\right\}_{a \in \mathfrak{a}}$ is an abelian subalgebra in $s l_{-}$commuting with $\mathfrak{a}$. Set $\mathfrak{a}_{S}:=\left\{a^{S}\right\}_{a \in \mathfrak{a}}$.

Consider the complexification $\mathfrak{b}^{c}:=\mathfrak{b} \otimes \mathbb{C}$ and $\mathbb{C}$-linearly extend the endomorphisms $\{\rho(a)\}_{a \in \mathfrak{a}}$ and $\sigma$. Also consider the complex Lie algebra $\operatorname{sl}\left(\mathfrak{b}^{c}\right)=\operatorname{sl}(\mathfrak{b}) \otimes \mathbb{C}$ and $\mathbb{C}$-linearly extend to $\operatorname{sl}\left(\mathfrak{b}^{c}\right)$ the involution $\Sigma$.

Let

$$
\mathfrak{b}^{c}=: \bigoplus_{\alpha \in \Phi} \mathfrak{b}_{\alpha}
$$

be the weight space decomposition with respect to the action of $\mathfrak{a}_{S}$. Note that for all $\alpha$, one has $\mathfrak{a}_{N} \cdot \mathfrak{b}_{\alpha} \subset \mathfrak{b}_{\alpha}$. Moreover, for all $X_{\alpha} \in \mathfrak{b}_{\alpha}$ and $a^{S} \in \mathfrak{a}_{S}$, one has

$$
\sigma\left(a^{S} . X_{\alpha}\right)=\alpha\left(a^{S}\right) \sigma\left(X_{\alpha}\right)=\sigma a^{S} \sigma^{-1} \sigma X_{\alpha}=\Sigma\left(a^{S}\right) . \sigma\left(X_{\alpha}\right)=-a^{S} . \sigma\left(X_{\alpha}\right) .
$$

Therefore, $-\alpha \in \Phi$ and $\sigma \mathfrak{b}_{\alpha}=\mathfrak{b}_{-\alpha}$. Note in particular that $\sigma \mathfrak{b}_{0}=\mathfrak{b}_{0}$.

Lemma 2.4. If the triple $t^{1}$ is assumed indecomposable and nonflat, then

$$
\mathfrak{b}_{0}=0 \text {. }
$$

Proof. Assume $0 \in \Phi$. For all $\alpha \in \Phi$, the subspace

$$
V_{\alpha}:=\mathfrak{b}_{\alpha} \oplus \mathfrak{b}_{-\alpha}
$$

of $\mathfrak{b}^{c}$ is stable under $\sigma$. In particular, the complexified involutive Lie algebra $\left(\mathfrak{g}^{c}, \sigma\right)$, where $\mathfrak{g}^{c}:=\mathfrak{g} \otimes \mathbb{C}$, can be expressed as $\mathfrak{g}^{c}=\mathfrak{a}^{c} \times{ }_{\rho} \mathfrak{b}^{c}$ with

$$
\mathfrak{b}^{c}=\bigoplus_{\alpha \in \Phi^{+}} \mathfrak{b}_{\alpha} \oplus \mathfrak{b}_{0}
$$

where the positive system of weights $\Phi^{+}$is chosen so that

$$
\Phi=\{0\} \cup \Phi^{+} \cup\left(-\Phi^{+}\right)
$$

(disjoint union). One therefore has the decomposition

$$
V_{\alpha}=\mathfrak{k}_{\alpha} \oplus \mathfrak{l}_{\alpha}
$$

into ( \pm )-eigenspaces for $\sigma$. Moreover, since $\mathfrak{g}^{c}=\left[\mathfrak{g}^{c}, \mathfrak{g}^{c}\right]$ and $\left[\mathfrak{a}, \mathfrak{b}_{\alpha}\right] \subset \mathfrak{b}_{\alpha}$, one has

$$
\mathfrak{l}_{\alpha}=\left[\mathfrak{a}, \mathfrak{k}_{\alpha}\right] \quad \text { and } \quad \mathfrak{k}_{\alpha}=\left[\mathfrak{a}, \mathfrak{l}_{\alpha}\right] \text {, }
$$

for all $\alpha \in \Phi^{+} \cup\{0\}$. This implies $\mathfrak{l}_{0}=\left[\mathfrak{a}_{N}, \mathfrak{k}_{0}\right]$ and $\mathfrak{k}_{0}=\left[\mathfrak{a}_{N}, \mathfrak{l}_{0}\right]$. Hence $\mathfrak{l}_{0}=$ $\left[\mathfrak{a}_{N},\left[\mathfrak{a}_{N}, \mathfrak{l}_{0}\right]\right]$ and an induction yields $\mathfrak{l}_{0}=0$.

Corollary 2.5. A nilpotent HI split symplectic symmetric space is flat. 
Proposition 2.6. Let $t=\left(\mathfrak{g}=\mathfrak{b} \times{ }_{\rho} \mathfrak{a}, \sigma, \Omega=\delta \xi\right)$ be the exact triple associated with a nonflat indecomposable split symplectic triple $t^{1}$. Let $\Phi$ be the set of weights associated with the (complex) action of $\mathfrak{a}_{S}$ on $\mathfrak{b}^{c}$. Fix a positive system $\Phi^{+}$and set

$$
\mathfrak{b}^{+}:=\bigoplus_{\alpha \in \Phi^{+}} \mathfrak{b}_{\alpha}
$$

Then the pair $\left(\mathfrak{s}^{c}:=\mathfrak{a}^{c} \times_{\rho} \mathfrak{b}^{+},\left.\Omega\right|_{\mathfrak{s}^{c}}\right)$ is a (complex) symplectic Lie algebra.

Proof. By the proof of Lemma 2.4, the restricted projection $\mathfrak{b}^{+} \stackrel{p}{\rightarrow} \mathfrak{l}^{c}$ mapping $X$ to $\frac{1}{2}(X-\sigma(X))$ is a linear isomorphism. Moreover, for all $X \in \mathfrak{b}^{+}, a \in \mathfrak{a}^{c}$, one has $\Omega(X, a)=\xi[p(c), a]$. The proposition follows from the nondegeneracy of the pairing $\mathfrak{a}^{c} \times \mathfrak{l}^{c} \rightarrow \mathbb{C}$.

Definition 2.5. Let $t$ be an HI split symplectic triple. Decompose $t$ into a direct sum of indecomposables and a flat factor. Proposition 2.6 then canonically associates to $t$ a (complex) symplectic Lie algebra $\mathfrak{s}^{c}(t)$, the complex symplectic Lie algebra associated with $t$.

Definition 2.6. A symplectic Lie algebra $(\mathfrak{s}, \omega)$ is called elementary solvable if

(i) it is a split extension of abelian Lie algebras $\mathfrak{a}$ and $\mathfrak{d}$ :

$$
0 \longrightarrow \mathfrak{d} \longrightarrow \mathfrak{s} \longrightarrow \mathfrak{a} \longrightarrow 0
$$

(ii) The cocycle $\omega$ is exact.

Conversely to Proposition 2.6, one has

Proposition 2.7. Every elementary solvable symplectic Lie algebra is associated with a split HI symplectic symmetric space.

Proof. Denote by $\rho: \mathfrak{a} \rightarrow \operatorname{End}(\mathfrak{d})$ the splitting homomorphism and by $\bar{\rho}: \mathfrak{a} \rightarrow$ End(d) the opposite representation: $\bar{\rho}(a)(X):=-\rho(a)(X), \quad X \in \mathfrak{d}$. Set

$$
\mathfrak{b}:=\mathfrak{d} \oplus \mathfrak{d}
$$

and let $\mathfrak{a}$ act on $\mathfrak{b}$ via $\rho \oplus \bar{\rho}$. Define the involution $\sigma_{\mathfrak{b}}$ of $\mathfrak{b}$ by

$$
\sigma_{\mathfrak{b}}(X, Y)=(Y, X), \quad X, Y \in \mathfrak{d} .
$$

Set

$$
\mathfrak{g}:=\mathfrak{b} \times{ }_{\rho \oplus \bar{\rho}} \mathfrak{a}
$$

and define the involution $\sigma$ of $\mathfrak{g}$ as

$$
\sigma:=\sigma_{\mathfrak{b}} \oplus\left(-\mathrm{id}_{\mathfrak{a}}\right) .
$$

One then observes that $(\mathfrak{g}, \sigma)$ is an involutive Lie algebra. We have $\mathfrak{k}=\{(X, X)\}_{X \in \mathfrak{d}}$ while $\mathfrak{p}=\{(X,-X)\}_{X \in \mathfrak{d}}$. 
Let $\eta \in \mathfrak{d}^{\star}$ be such that $\delta \eta=\omega$ and define $\xi \in \mathfrak{k}^{\star}$ by

$$
\xi(X, X):=\eta(X), \quad X \in \mathfrak{d} .
$$

Extending $\xi$ to $\mathfrak{g}$ by 0 on $\mathfrak{p}$, one defines a symplectic coboundary on $\mathfrak{g}$ :

$$
\Omega:=\delta \xi
$$

The triple $(\mathfrak{g}, \sigma, \Omega)$ then defines the desired elementary solvable symplectic symmetric space.

\section{Strict deformation quantization for proper actions}

We first recall that to an involutive Lie algebra, one associates a simply connected symmetric space $M$ in the usual way (see [Kobayashi and Nomizu 1969], for example). When associated with a symplectic triple, the space $M$ turns out to be naturally endowed with an invariant symplectic structure. The space $M$ is then called a symplectic symmetric space. One then has

Theorem 3.1 [Bieliavsky 2002]. Let $M$ be the simply connected symplectic symmetric space associated with an HI split symplectic triple. Assume $M$ is strictly geodesically convex with respect to its canonical affine connexion. There is a oneparameter family $\left\{K_{\theta} \in C^{\infty}(M \times M \times M, \mathbb{C})\right\}_{\theta \in \mathbb{R}}$ of smooth invariant three-point kernels, and a corresponding family $\left\{\mathscr{H}_{\theta} \in C^{\infty}(M)\right\}_{\theta}$ of invariant function spaces, such that

(i) $\mathscr{D}(M):=C_{c}^{\infty}(M) \subset \mathscr{H}_{\theta}$ for all $\theta$;

(ii) the formula

$$
u \star_{\theta} v(x) ;=\int_{M \times M} K(x, y, z) u(y) v(z) \mathrm{d} y \mathrm{~d} z \quad(u, v \in \mathscr{D}(M))
$$

extends to $\mathscr{H}_{\theta}$ as an associative $\mathbb{C}$-algebra product law denoted hereafter $\star_{\theta}$, and the automorphism group of $M$ acts on the algebra $\left(\mathscr{H}_{\theta}, \star_{\theta}\right)$ by algebra automorphisms;

(iii) for $u, v \in \mathscr{D}(M)$ and $x \in M$, one has the asymptotic expansion

$$
u \star_{\theta} v(x) \sim u v(x)+\frac{\theta}{2 i}\{u, v\}(x)+o\left(\theta^{2}\right),
$$

where $\{$,$\} denotes the invariant symplectic Poisson bracket on C^{\infty}(M)$.

We now pass to the announced nonformal UDFs. In this section, $(\mathfrak{s}, \Omega)$ denotes an elementary solvable symplectic Lie algebra associated with a strictly geodesically convex HI split symplectic symmetric space $M$. We denote by $\mathbb{S}$ the corresponding simply connected symplectic Lie group, whose identity element we write as $e$. 
Now consider a proper smooth action of $\mathbb{S}$ on a manifold $X$ :

$$
\mathbb{S} \times X \rightarrow X:(g, x) \mapsto \tau_{g}(x) .
$$

And denote by

$$
\mathbb{S} \times C^{\infty}(X) \rightarrow C^{\infty}(X):(g, a) \mapsto \alpha_{g}(a)
$$

the corresponding left action on functions on $X$. At last, for all $a \in C^{\infty}(X)$ and $x \in X$, set

$$
\alpha^{x}(a): \mathbb{S} \rightarrow \mathbb{C}: g \mapsto \alpha_{g}(a)(x):=\tau_{g^{-1}}^{\star}(a) .
$$

Theorem 3.2. Define the space

$$
\mathbb{B}_{\theta} ;=\left\{a \in C^{\infty}(X) \text { such that } \alpha^{x}(a) \in \mathscr{H}_{\theta} \text { for all } x \in X\right\} .
$$

Then

(i) $\mathscr{D}(X):=C_{c}^{\infty}(X) \subset \mathbb{B}_{\theta}$ for all $\theta$, and

(ii) the formula

$$
a \star_{\theta}^{X} b(x):=\left(\alpha^{x}(a) \star_{\theta} \alpha^{x}(b)\right)(e)
$$

defines an associative $\mathbb{C}$-algebra product law on the function space $\mathbb{B}_{\theta}$.

Proof. Item (i) follows from the properness of the action and item (i) of the preceding theorem. Item (ii) follows from [Bieliavsky 2002, p. 282, item (ii)]; see also [Bieliavsky et al. 2003].

For more general actions, we shall remain at the formal level. We shall prove in the next section that the asymptotic expansion (5) actually defines an invariant formal star product on $M$. For simplicity, we shall treat only the case $\operatorname{dim} \mathbb{S}=2$ but as it will appear this is not essential.

\section{Formal universal deformation formulae}

We present the $a x+b$ group as $\mathbb{S}=\mathbb{R}^{2}=\{(a, \ell)\}$ with multiplication law

$$
(a, \ell) .\left(a^{\prime}, \ell^{\prime}\right):=\left(a+a^{\prime}, e^{-2 a^{\prime}} \ell+\ell^{\prime}\right) .
$$

The Lie algebra $\mathfrak{s}$ of $\mathbb{S}$ is then realized as $\mathfrak{s}=\operatorname{span}\{H, E\}$ with

$$
(a, 0)=: \exp (a H) \text { and }(0, \ell)=: \exp (\ell E) .
$$

For $X \in \mathfrak{g}$, we denote by $\tilde{X}$ the corresponding left-invariant vector field on $\mathbb{S}$.

We denote by $\mathscr{S}$ the Schwartz space on $\mathbb{S}=\{(a, \ell)\}$. For $u \in \mathscr{Y}$, we denote by $\mathscr{F}(u)$ the partial Fourier transform

$$
\mathscr{F}(u)(a, \xi):=: \hat{u}(a, \xi):=: \int e^{-i \xi \ell} u(a, \ell) \mathrm{d} \ell .
$$


We set $\tilde{S}:=\{(a, \xi)\}$ and we denote by $\tilde{\mathscr{S}}$ the space of Schwartz functions in these variables. Thus, one has the isomorphism $\mathscr{F}: \mathscr{Y} \rightarrow \tilde{\mathscr{S}}$. For $\theta \in \mathbb{R}$, we define the following diffeomorphism $\phi_{\theta}: \tilde{\mathbb{S}} \rightarrow \tilde{\mathbb{S}}$ :

$$
\phi_{\theta}(a, \xi):=\left\{\begin{array}{cc}
\left(a, \frac{1}{2 \theta} \sinh (2 \theta \xi)\right) & \text { for } \theta \neq 0, \\
(a, \xi) & \text { for } \theta=0 .
\end{array}\right.
$$

Setting $\tau_{\theta}:=\mathscr{F}^{-1} \circ\left(\phi_{\theta}^{-1}\right)^{\star} \circ \mathscr{F}$, we formally define the following distribution space on $\mathbb{S}$ :

$$
\mathscr{E}_{\theta}:=\tau_{\theta}(\mathscr{Y}) .
$$

Lemma 4.1 [Bieliavsky 2002]. For all $\theta \in \mathbb{R}$,

$$
\mathscr{S} \subset \mathscr{E}_{\theta} \subset \mathscr{Y}^{\prime} \cap C^{\infty}(\mathbb{S})
$$

where $\mathscr{Y}^{\prime}$ denotes the space of tempered distributions in variables $(a, \ell)$.

\section{Lemma 4.2.}

$$
\left(\phi_{\theta}^{-1}\right)^{\star}(\tilde{\mathscr{S}})=\left\{\begin{array}{cc}
\left(\phi_{1 / 2}^{-1}\right)^{\star}(\tilde{\mathscr{S}}) & \text { for } \theta \neq 0, \\
\tilde{\mathscr{S}} & \text { for } \theta=0 .
\end{array}\right.
$$

Proof. First observe that $f \in\left(\phi_{\theta}^{-1}\right)^{\star} \tilde{\mathscr{Y}}$ if and only if for all $M, N \in \mathbb{N}$ one has $\int \xi^{M} \partial_{\xi}^{N}\left(\phi_{\theta}^{\star} f\right) \mathrm{d} \xi<\infty$. Let us restrict ourself to the case $N=1$, the general case being entirely similar. One has $\partial_{\xi}\left(\phi_{\theta}^{\star} f\right)(\xi)=\cosh (2 \theta \xi) \phi_{\theta}^{\star}\left(\partial_{\eta} f\right)=$ $\phi_{\theta}^{\star}\left(\sqrt{1+(2 \theta \eta)^{2}} \partial_{\eta} f\right)$. Therefore, the condition becomes

$$
\int \operatorname{arcsinh}(2 \theta \eta) \partial_{\eta} f(\eta) \mathrm{d} \eta<\infty
$$

Since the functions $\operatorname{arcsinh}(2 \theta \eta) \partial_{\eta} f(\eta)$ and $\operatorname{arcsinh} \eta \partial_{\eta} f(\eta)$ have, up to a $\theta$ dependant multiple, the same asymptotic behavior for large $|\eta|$, the finiteness of the above integral is equivalent to the finiteness of $\int \operatorname{arcsinh} \eta \partial_{\eta} f(\eta) \mathrm{d} \eta$.

In particular, for all $\theta \in \mathbb{R}$, one has

$$
\left(\phi_{\theta}^{-1}\right)^{\star}(\tilde{\mathscr{Y}}) \subset\left(\phi_{1 / 2}^{-1}\right)^{\star}(\tilde{\mathscr{Y}}) .
$$

The above lemma leads us to define the following spaces:

$$
\hat{\mathscr{E}}:=\left(\phi_{1 / 2}^{-1}\right)^{\star}(\tilde{\mathscr{Y}}) \text { and } \mathscr{E}:=\mathscr{F}^{-1}(\hat{\mathscr{E}}) \text {. }
$$

We then observe the following obvious fact.

Lemma 4.3. Denote by $\mathscr{B}_{\infty}(\tilde{\mathbb{S}})$ the space of smooth functions $w \in C^{\infty}(\tilde{\mathbb{S}})$ such that for all multi-index $\alpha, D^{\alpha} w \in C_{0}(\tilde{\mathbb{S}})$. Then,

$$
\hat{\mathscr{E}} \subset \mathscr{B}_{\infty}(\tilde{\mathbb{S}}) \quad \text { and } \quad \partial_{\xi}^{2}(\hat{\mathscr{E}}) \subset L^{1}(\tilde{S}) \text {. }
$$


Lemma 4.4. Let $u \in \mathscr{Y}$. Then,

(i) there exists a dense open subset $\Omega \subset G$ where, for all $\theta \in \mathbb{R}$, the tempered distribution $\tau_{\theta}(u)$ coincides with a smooth function, $\tau_{\theta}^{\text {loc }}(u) \in C^{\infty}(\Omega)$, in the sense that for all $\psi \in \mathscr{D}(\Omega),\left\langle\tau_{\theta}(u), \psi\right\rangle=\left\langle\tau_{\theta}^{\mathrm{loc}}(u), \psi\right\rangle$.

(ii) The function $\mathbb{R} \rightarrow C^{\infty}(\Omega): \theta \mapsto \tau_{\theta}^{\mathrm{loc}}(u)$ is smooth.

Proof. Abel's criterion implies that for $\ell \neq 0$, the improper integral $\tau_{\theta}^{\text {loc }}(u)(a, \ell):=$ $\frac{1}{2 \pi} \int e^{i \xi \ell}\left(\phi_{\theta}^{-1}\right)^{\star} \hat{u}(\xi) \mathrm{d} \xi$ converges, since, by Lemma $4.3,\left(\phi_{\theta}^{-1}\right)^{\star} \hat{u} \in C_{0}(\tilde{S})$. The function $\tau_{\theta}^{\text {loc }}(u)$ is smooth in $(\theta, x) \in \mathbb{R} \times \Omega$ where $\Omega:=\{(a, \ell) \mid \ell \neq 0\}$. Indeed, observing that $e^{i \xi \ell}=\frac{1}{i \ell} \partial_{\xi} e^{i \xi \ell}$, one obtains, using Lemma 4.3:

$$
\begin{aligned}
\tau_{\theta}^{\operatorname{loc}}(u)(a, \ell) & =\frac{i}{2 \pi \ell} \lim _{r \rightarrow \infty} \int_{-r}^{r} e^{i \xi \ell} \partial_{\xi}\left(\phi_{\theta}^{-1}\right)^{\star} \hat{u}(\xi) \mathrm{d} \xi \\
& =\frac{i}{2 \pi \ell} \int e^{i \xi \ell} \partial_{\xi}\left(\phi_{\theta}^{-1}\right)^{\star} \hat{u}(\xi) \mathrm{d} \xi .
\end{aligned}
$$

Hence, $\tau_{\theta}^{\mathrm{loc}}(u)(a, \ell)=\frac{-1}{2 \pi \ell^{2}} \int e^{i \xi \ell} \partial_{\xi}^{2}\left(\phi_{\theta}^{-1}\right)^{\star} \hat{u}(\xi) \mathrm{d} \xi$, which is an existing integral. Now, by Lemma 4.2 , one observes that setting $\hat{u}_{\theta}:=\phi_{1 / 2}^{\star}\left(\phi_{\theta}^{-1}\right)^{\star} \hat{u}$ defines a smooth family of Schwartz functions $\left\{\hat{u}_{\theta}\right\} \subset \tilde{\mathscr{S}}$ such that $\left(\phi_{\theta}^{-1}\right)^{\star} \hat{u}=\left(\phi_{1 / 2}^{-1}\right)^{\star} \hat{u}_{\theta}$. Therefore, one has

$$
\tau_{\theta}^{\mathrm{loc}}(u)(a, \ell)=\frac{-1}{2 \pi \ell^{2}} \int e^{i \xi \ell} \partial_{\xi}^{2}\left(\phi_{1 / 2}^{-1}\right)^{\star} \hat{u}_{\theta}(\xi) \mathrm{d} \xi,
$$

whose integrand is bounded by the integrable function $\left|\partial_{\xi}^{2}\left(\phi_{1 / 2}^{-1}\right)^{\star} \hat{u}_{\theta_{0}}\right|$ for $\theta \in[-\epsilon, \epsilon]$. One proceeds similarly for derivatives. It follows that $\tau_{\theta}^{\text {loc }}(u)$ defines an element of $C^{\infty}\left(\mathbb{R}, C^{\infty}(\Omega)\right)$ [Trèves 1967 , Theorem 40.1, p. 415]. Of course, $\left\langle\tau_{\theta}(u), \psi\right\rangle=$ $\left\langle\tau_{\theta}^{\text {loc }}(u), \psi\right\rangle$ for all $\psi \in \mathscr{D}(\Omega)$.

Proposition 4.5 [Bieliavsky 2002]. (i) The map $T_{\theta}:=\mathscr{F}^{-1} \circ \phi_{\theta}^{\star} \circ \mathscr{F}_{\text {establishes } a}$ linear isomorphism

$$
T_{\theta}: \mathscr{E}_{\theta} \rightarrow \mathscr{Y}
$$

(ii) Denote by $\star_{\theta}^{0}$ Weyl's product on $\mathscr{Y}$. The formula

$$
a \star_{\theta} b:=\tau_{\theta}\left(T_{\theta} a \star_{\theta}^{0} T_{\theta} b\right) ; \quad a, b \in \mathscr{E}_{\theta}
$$

defines an associative product $\star_{\theta}$ on $\mathscr{E}_{\theta}$.

(iii) At the level of smooth compactly supported $u, v \in C_{c}^{\infty}(\mathbb{S})$ and for all $x_{0} \in \Omega$, one has the oscillatory integral formula

$$
\begin{aligned}
& u \star_{\theta} v\left(x_{0}\right)= \\
& \frac{1}{4 \pi^{2} \theta^{2}} \int_{G \times G} \cosh 2\left(a_{1}-a_{2}\right) \exp \frac{i \underset{0,1,2}{\Psi} \ell_{2} \sinh 2\left(a_{0}-a_{1}\right)}{2 \theta} u\left(x_{1}\right) v\left(x_{2}\right) \mathrm{d} x_{1} \mathrm{~d} x_{2},
\end{aligned}
$$

where $x_{j}=\left(a_{j}, \ell_{j}\right), j=0,1,2$. 
(iv) The three-point kernel

$$
K_{\theta}\left(x_{0}, x_{1}, x_{2}\right):=\frac{1}{4 \pi^{2} \theta^{2}} \cosh \left(2\left(a_{1}-a_{2}\right)\right) e^{\frac{i}{2 \theta} \underset{0,1,2}{\leftrightarrows} \sinh \left(2\left(a_{0}-a_{1}\right)\right) \ell_{2}}
$$

is left-invariant with respect to the diagonal action of $\mathbb{S}$ on $G \times G \times G$.

Remark 4.1. The formula in (iii) holds at every point $x_{0} \in G$. Indeed, by leftinvariance of the kernel, one has for every choice of $y \in \Omega: u \star_{\theta} v\left(x_{0}\right)=\left(L_{x_{0} y^{-1}}^{\star} u \star_{\theta}\right.$ $\left.L_{x_{0} y^{-1}}^{\star} v\right)(y)$.

We set, for integral $n \geq 1$,

$$
\beta(2 n+1):=\frac{1}{2} \frac{(-1)^{n}}{4^{n}} \frac{1}{2 n+1} \frac{(2 n) !}{(n !)^{2}}=\frac{(-1)^{n}}{4^{n}} \frac{1}{2 n+1}\left(\begin{array}{c}
2 n-1 \\
n-1
\end{array}\right),
$$

and further $\beta(1):=\frac{1}{2}$ and $\beta(2 n):=0$ for $n \geq 0$.

Definition 4.1. Let $k$ and $N$ be positive integers such that $k \leq N$. We set

$$
B_{N}^{k}:=\sum_{\substack{k_{1}, \ldots, k_{N} \\ k=k_{1}+\cdots+k_{N} \\ N=1 k_{1}+2 k_{2}+\cdots+N k_{N}}} \frac{N !}{k_{1} ! k_{2} ! \cdots k_{N} !} \beta(1)^{k_{1}} \beta(2)^{k_{2}} \cdots \beta(N)^{k_{N}},
$$

with the convention that $\beta(2 n)^{0}:=1$ and $B_{0}^{0}:=1$.

Lemma 4.6. Let $u \in C^{\infty}(\mathbb{S})$ and $N \in \mathbb{N}_{0}$. Then

$$
\left.\frac{\mathrm{d}^{N}}{\mathrm{~d} \theta^{N}}\right|_{\xi=0}\left(R_{\left(\frac{1}{2} \operatorname{arcsinh} \xi, 0\right)}^{\star} u\right)=\sum_{k=1}^{N} B_{N}^{k} \widetilde{H}^{k} \cdot u,
$$

where $R$ denotes right multiplication on $\mathbb{S}$.

Proof. We shall use Faà di Bruno's formula [1857], which computes the higher order derivatives of composed functions:

$$
\begin{aligned}
& \frac{d^{n}}{d \xi^{n}}[R(b(\xi))] \\
& \quad=\sum \frac{n !}{k_{1} ! k_{2} ! \cdots k_{n} !}\left(\frac{b^{(1)}(\xi)}{1 !}\right)^{k_{1}}\left(\frac{b^{(2)}(\xi)}{2 !}\right)^{k_{2}} \cdots\left(\frac{b^{(n)}(\xi)}{n !}\right)^{k_{n}} R^{(k)}(b(\xi)),
\end{aligned}
$$

with $k:=k_{1}+\cdots+k_{n}$ and where $\left(k_{1}, \ldots, k_{n}\right)$ runs over all partitions of $n$ (solutions of the equation $n=1 k_{1}+2 k_{2}+\cdots+n k_{n}$ ). We now observe the Taylor expansion $\frac{1}{2} \operatorname{arcsinh} \xi=\frac{1}{2} \xi+\sum_{n \geq 1} \frac{(-1)^{n}}{4^{n}} \frac{1}{2 n+1}\left(\begin{array}{c}2 n-1 \\ n-1\end{array}\right) \xi^{2 n+1}=\sum_{n \geq 0} \frac{(-1)^{n}}{2 \cdot 4^{n}} \frac{1}{2 n+1} \frac{(2 n) !}{(n !)^{2}} \xi^{2 n+1}$.

Plugging $b(\xi):=\frac{1}{2} \operatorname{arcsinh} \xi$ and $R(a):=R_{(a, 0)}^{\star} u(x)$ into (6) at $\xi=0$ then yields the lemma. 
Lemma 4.7. Let $\gamma$ and $\mu$ be maps from $\mathbb{R} \times \mathscr{Y}$ to $C^{\infty}(\mathbb{S})$ such that

(i) for every $u \in \mathscr{S}$ the functions $\gamma(., u)$ and $\mu(., u)$ belong to $C^{\infty}\left(\mathbb{R}, C^{\infty}(\mathbb{S})\right)$;

(ii) for all $\theta$ the partial functions $\gamma(\theta,$.$) and \mu(\theta,$.$) are \mathbb{C}$-linear.

Assume the function

$$
\theta \mapsto \phi(\theta, u):=\mu(\theta, \gamma(\theta, u))
$$

to be an element of $C^{\infty}\left(\mathbb{R}, C^{\infty}(\mathbb{S})\right)$ as well. Denoting by

$$
\gamma(\theta, u) \sim \sum_{m} \theta^{m} \Gamma_{m}(u), \quad \mu(\theta, u) \sim \sum_{m} \theta^{m} M_{m}(u), \quad \phi(\theta, u) \sim \sum_{m} \theta^{m} \Phi_{m}(u)
$$

the corresponding Taylor expansions, one has

$$
\Phi_{m}(u)=\sum_{m=n+k} M_{k}\left(\Gamma_{n}(u)\right)
$$

Proof. Let $R_{N}^{\mu}(u)$ be the rest at order $N$ in the Taylor expansion of $\mu$ [Bourbaki 1967]. Then,

$$
\left[\theta \mapsto R_{N}^{\mu}(\gamma(\theta, u))\right] \in o\left(\theta^{N}\right) .
$$

Indeed, $R_{N}^{\gamma}(u)=\theta^{N} \rho_{N}(u)$ where $\rho^{N}(u)$ is continuous in a neighborhood of $\theta=0$. Therefore $\theta^{-(N+1)} R_{N}^{\mu}(\gamma(\theta, u))=\sum_{k}^{N+1} \theta^{k-N-1} R_{N}^{\mu}\left(\Gamma_{k}(u)\right)+\theta R_{N}^{\mu}\left(\rho_{N+1}(u)\right)$. The first $N+2$ terms of this sum tend to 0 with $\theta$. In the last term, one observes that $R_{N}^{\mu}\left(\rho_{N+1}(u)\right)$ is continuous and hence bounded for $\theta \in[-\epsilon, \epsilon]$, which proves the assertion and with it the lemma.

Of course an analogous statement holds for bilinear maps.

Lemma 4.8. For every smooth compactly supported $u, v \in C_{c}^{\infty}(\mathbb{S})$, the functions of $\theta$ given by $T_{\theta}(u), u \star_{\theta}^{0} v$ and $u \star_{\theta} v$ belong to $C^{\infty}\left(\mathbb{R}, C^{\infty}(\mathbb{S})\right)$.

Proof. For every $x_{0} \in G$,

$$
T_{\theta}(u)\left(x_{0}\right)=\frac{1}{2 \pi} \int e^{i \ell_{0} \xi} \phi_{\theta}^{\star} \hat{u}\left(a_{0}, \xi\right) \mathrm{d} \xi .
$$

The integrand $e^{i \ell_{0} \xi} \phi_{\theta}^{\star} \hat{u}\left(a_{0}, \xi\right)$ is smooth in the variables $\left(a_{0}, \ell_{0}, \theta\right)$ as well as every of its partial derivatives. Moreover, each of these functions is bounded by an integrable function since $\phi_{\theta}^{\star} \hat{u}$ is Schwartz. Therefore the function $\left(x_{0}, \theta\right) \mapsto T_{\theta}(u)\left(x_{0}\right)$ is smooth.

The argument is similar for the other functions, simpler in fact since the integrand is in both cases compactly supported.

Theorem 4.9. For every smooth compactly supported $u, v \in C_{c}^{\infty}(\mathbb{S})$ and for every $x \in G$, the function $\left[\mathbb{R} \rightarrow \mathbb{C}: \theta \mapsto u \star_{\theta} v(x)\right]$ is smooth. Its Taylor series at 0 defines 
an associative formal star product $\star_{\nu}$ on $C^{\infty}(\mathbb{S}) \llbracket v \rrbracket$ whose explicit expression is

$$
\begin{aligned}
u \star_{v} v:=u v+ & \sum_{n=1}^{\infty} \frac{v^{n}}{n !}(-2 i)^{n} \sum_{k=0}^{n}(-1)^{k+1}\left(\begin{array}{l}
n \\
k
\end{array}\right) \\
& \times\left(\left(\sum_{m=0}^{k} B_{k}^{m} \widetilde{H}^{m} \widetilde{E}^{n-k} \cdot v\right)\left(\sum_{m=0}^{n-k} B_{n-k}^{m} \widetilde{H}^{m} \widetilde{E}^{k} \cdot u\right)\right. \\
& -2\left(\sum_{r=0}^{k}\left(\begin{array}{l}
k \\
r
\end{array}\right)(k-r) \beta(k-r) \sum_{p=0}^{r} B_{r}^{p} \widetilde{H}^{p} \widetilde{E}^{n-k} . v\right) \\
& \left.\times\left(\sum_{s=0}^{n-k}\left(\begin{array}{c}
n-k \\
s
\end{array}\right)(n-k-s) \beta(n-k-s) \sum_{q=0}^{s} B_{s}^{q} \widetilde{H}^{q} \widetilde{E}^{k} \cdot u\right)\right)
\end{aligned}
$$

Proof. Using left invariance one gets

$$
u \star_{\theta} v\left(x_{0}\right)=\left.\left.\int K_{\theta}\left(0, x_{1}, x_{2}\right) R_{x_{1}}^{\star} u\right|_{x_{0}} R_{x_{2}}^{\star} v\right|_{x_{0}} d x_{1} d x_{2} .
$$

Making the change of variables $\xi_{i}:=\sinh \left(2 a_{i}\right), \eta_{i}:=\frac{1}{2 \theta} \ell_{i}$ yields

$u \star_{\theta} v\left(x_{0}\right)=\frac{1}{4 \pi^{2}} \int\left(1-\gamma\left(\xi_{1}\right) \gamma\left(\xi_{2}\right)\right) e^{-i\left(\xi_{1} \eta_{2}-\xi_{2} \eta_{1}\right)}$ $\times\left.\left. r_{\left(\xi_{1}, 2 \theta \eta_{1}\right)}^{\star} u\right|_{x_{0}} r_{\left(\xi_{2}, 2 \theta \eta_{2}\right)}^{\star} v\right|_{x_{0}} \mathrm{~d} \xi_{1} \mathrm{~d} \xi_{2} \mathrm{~d} \eta_{1} \mathrm{~d} \eta_{2}$,

where $\gamma(\xi):=\xi / \sqrt{1+\xi^{2}}$ and $\left.r_{(\xi, 2 \theta \eta)}^{\star} u\right|_{x}:=u\left(a+\frac{1}{2} \operatorname{arcsinh} \xi, e^{-\operatorname{arcsinh} \xi} \ell+2 \theta \eta\right)$. Now observe the asymptotic expansion $\left(\theta_{0} \neq 0\right)$

$$
\begin{aligned}
& u \star_{\theta} v\left(x_{0}\right)=\sum_{n=0}^{N} \frac{\left(\theta-\theta_{0}\right)^{n}}{n !} \frac{1}{4 \pi^{2}} \int\left(1-\gamma\left(\xi_{1}\right) \gamma\left(\xi_{2}\right)\right) e^{-i\left(\xi_{1} \eta_{2}-\xi_{2} \eta_{1}\right)} \\
& \times\left.\frac{\mathrm{d}^{n}}{\mathrm{~d} \theta^{n}}\left[\left.\left.r_{\left(\xi_{1}, 2 \theta \eta_{1}\right)}^{\star} u\right|_{x_{0}} r_{\left(\xi_{2}, 2 \theta \eta_{2}\right)}^{\star} v\right|_{x_{0}}\right]\right|_{\theta=\theta_{0}} \mathrm{~d} \xi_{1} \mathrm{~d} \xi_{2} \mathrm{~d} \eta_{1} \mathrm{~d} \eta_{2} \\
&+o\left(\left(\theta-\theta_{0}\right)^{N}\right) .
\end{aligned}
$$

One has

$$
\begin{aligned}
\frac{\mathrm{d}^{n}}{\mathrm{~d} \theta^{n}}\left[\left.r_{\left(\xi_{1}, 2 \theta \eta_{1}\right)}^{\star} u\right|_{x_{0}}\right. & \left.\left.r_{\left(\xi_{2}, 2 \theta \eta_{2}\right)}^{\star} v\right|_{x_{0}}\right]\left.\right|_{\theta=\theta_{0}} \\
& =\left.\left.\sum_{k=0}^{n}\left(\begin{array}{c}
n \\
k
\end{array}\right) \frac{\mathrm{d}^{k}}{\mathrm{~d} \theta^{k}}\left[\left.r_{\left(\xi_{1}, 2 \theta \eta_{1}\right)}^{\star} u\right|_{x_{0}}\right]\right|_{\theta=\theta_{0}} \frac{\mathrm{d}^{n-k}}{\mathrm{~d} \theta^{n-k}}\left[\left.r_{\left(\xi_{2}, 2 \theta \eta_{2}\right)}^{\star} v\right|_{x_{0}}\right]\right|_{\theta=\theta_{0}} \\
& =\left.\left.2^{n} \sum_{k=0}^{n}\left(\begin{array}{l}
n \\
k
\end{array}\right) \eta_{1}^{k} \eta_{2}^{n-k} r_{\left(\xi_{1}, 2 \theta_{0} \eta_{1}\right)}^{\star}\left(\widetilde{E}^{k} \cdot u\right)\right|_{x_{0}} r_{\left(\xi_{2}, 2 \theta_{0} \eta_{2}\right)}^{\star}\left(\widetilde{E}^{n-k} \cdot v\right)\right|_{x_{0}},
\end{aligned}
$$


where $\exp (a H):=(a, 0)$ and $\exp (\ell E):=(0, \ell)$. Therefore, an integration by parts yields (for $n \geq 1$ )

$$
\begin{aligned}
& \left.\int\left(1-\gamma\left(\xi_{1}\right) \gamma\left(\xi_{2}\right)\right) e^{-i\left(\xi_{1} \eta_{2}-\xi_{2} \eta_{1}\right)} \frac{\mathrm{d}^{n}}{\mathrm{~d} \theta^{n}}\left[\left.\left.r_{\left(\xi_{1}, 2 \theta \eta_{1}\right)}^{\star} u\right|_{x_{0}} r_{\left(\xi_{2}, 2 \theta \eta_{2}\right)}^{\star} v\right|_{x_{0}}\right]\right|_{\theta=\theta_{0}} \mathrm{~d} \xi_{1} \mathrm{~d} \xi_{2} \mathrm{~d} \eta_{1} \mathrm{~d} \eta_{2} \\
& =(-2 i)^{n} \sum_{k=0}^{n}(-1)^{k}\left(\begin{array}{c}
n \\
k
\end{array}\right)\left(\int e^{-i\left(\xi_{1} \eta_{2}-\xi_{2} \eta_{1}\right)}\right. \\
& \quad \times\left(\left.\left.\partial_{\xi_{2}}^{k} r_{\left(\xi_{2}, 2 \theta_{0} \eta_{2}\right)}^{\star}\left(\widetilde{E}^{n-k} \cdot v\right)\right|_{x_{0}} \partial_{\xi_{1}}^{n-k} r_{\left(\xi_{1}, 2 \theta_{0} \eta_{1}\right)}^{\star}\left(\widetilde{E}^{k} \cdot u\right)\right|_{x_{0}}\right. \\
& \left.\left.\quad-\partial_{\xi_{2}}^{k}\left[\left.\gamma\left(\xi_{2}\right) r_{\left(\xi_{2}, 2 \theta_{0} \eta_{2}\right)}^{\star}\left(\widetilde{E}^{n-k} \cdot v\right)\right|_{x_{0}}\right] \partial_{\xi_{1}}^{n-k}\left[\left.\gamma\left(\xi_{1}\right) r_{\left(\xi_{1}, 2 \theta_{0} \eta_{1}\right)}^{\star}\left(\widetilde{E}^{k} \cdot u\right)\right|_{x_{0}}\right]\right)\right) .
\end{aligned}
$$

By observing that $\left.\lim _{\eta \rightarrow 0} \partial_{\xi} r_{(\xi, \eta)}^{\star} u\right|_{x}=\left.\partial_{\xi} r_{(\xi, 0)}^{\star} u\right|_{x}$, one gets

$$
\begin{aligned}
\lim _{\theta \rightarrow 0} \int \gamma\left(\xi_{1}\right) \gamma\left(\xi_{2}\right) e^{-i\left(\xi_{1} \eta_{2}-\xi_{2} \eta_{1}\right)} \frac{\mathrm{d}^{n}}{\mathrm{~d} \theta^{n}}\left[\left.\left.r_{\left(\xi_{1}, 2 \theta \eta_{1}\right)}^{\star} u\right|_{x_{0}} r_{\left(\xi_{2}, 2 \theta \eta_{2}\right)}^{\star} v\right|_{x_{0}}\right] \mathrm{d} \xi_{1} \mathrm{~d} \xi_{2} \mathrm{~d} \eta_{1} \mathrm{~d} \eta_{2} \\
=\left.4 \pi^{2}(-2 i)^{n} \sum_{k=0}^{n}(-1)^{k+1}\left(\begin{array}{l}
n \\
k
\end{array}\right) \partial_{\xi}^{k}\left[\left.\gamma(\xi) r_{(\xi, 0)}^{\star}\left(\widetilde{E}^{n-k} \cdot v\right)\right|_{x_{0}}\right]\right|_{\xi=0} \\
\times\left.\partial_{\xi}^{n-k}\left[\left.\gamma(\xi) r_{(\xi, 0)}^{\star}\left(\widetilde{E}^{k} \cdot u\right)\right|_{x_{0}}\right]\right|_{\xi=0},
\end{aligned}
$$

which, by using Lemma 4.6 and the expansion

$$
\gamma(\xi)=\xi+\sum_{m \geq 1} \frac{(-1)^{m}}{4^{m}} \frac{m+1}{m}\left(\begin{array}{c}
2 m \\
m+1
\end{array}\right) \xi^{2 m+1}=\sum_{m \geq 0} \frac{(-1)^{m}}{4^{m}} \frac{(2 m) !}{(m !)^{2}} \xi^{2 m+1}
$$

is seen to be

$$
\begin{aligned}
4 \pi^{2}(-2 i)^{n} \sum_{k=0}^{n}(-1)^{k+1}\left(\begin{array}{l}
n \\
k
\end{array}\right) & \left(\left.\sum_{r=0}^{k}\left(\begin{array}{l}
k \\
r
\end{array}\right) \gamma^{(k-r)}(0) \sum_{p=0}^{r} B_{r}^{p}\left(\widetilde{H}^{p} \widetilde{E}^{n-k} \cdot v\right)\right|_{x_{0}}\right) \\
& \left.\times\left(\left.\sum_{s=0}^{n-k}\left(\begin{array}{c}
n-k \\
s
\end{array}\right) \gamma^{(n-k-s)}(0) \sum_{q=0}^{s} B_{s}^{q}\left(\widetilde{H}^{q} \widetilde{E}^{k} \cdot u\right)\right|_{x_{0}}\right)\right) .
\end{aligned}
$$

This leads to the announced asymptotic expansion where $v$ is the formal parameter corresponding to $\theta$.

Regarding associativity, one first observes that for all $x_{0} \in \Omega$, one has the coincidence: $u \star_{\theta} v\left(x_{0}\right)=\tau_{\theta}^{\text {loc }}\left(T_{\theta} u \star_{\theta}^{0} T_{\theta} v\right)\left(x_{0}\right)$. Note that, independently, the left hand side is smooth in $\theta$ by virtue of Lemma 4.8 , while the right hand side is smooth as well by virtue of Lemmas 4.4, 4.7 and 4.8. Lemma 4.7 therefore identifies the above-computed Taylor coefficients with the cochains of the star product obtained by intertwining Moyal's star product with a formal equivalence. 
Remark 4.2. Our construction differs radically from the (formal) universal deformation formula of Giaquinto and Zhang [1998]: It was shown in [Bieliavsky et al. 2005b] that the (maximal) invariance diffeomorphism group of GiaquintoZhang star product on $a x+b$ is isomorphic to $\operatorname{Sp}(1, \mathbb{R}) \times \mathbb{R}^{2}$, while in our case the corresponding maximal invariance group is the automorphism group of the underlying symplectic symmetric space - in the two-dimensional case, the solvable group $\mathrm{SO}(1,1) \times \mathbb{R}^{2}$.

\section{Hopf structure}

The formal star product $\star_{v}$ given by formula (7) can be described as an example of the following construction [Bieliavsky et al. 2005a].

Definition 5.1 [Bonneau and Sternheimer 2005]. Let $B$ be a cocommutative bialgebra and $C$ a $B$-bimodule algebra. The $L$-R-smash product $C \backsim B$ is the algebra constructed on the vector space $C \otimes B$ where the multiplication is defined by

$$
(f \otimes a) \star(g \otimes b)=\sum_{(a)(b)}\left(f<b_{(1)}\right)\left(a_{(1)} \rightarrow g\right) \otimes a_{(2)} b_{(2)}
$$

for $f, g \in C$ and $a, b \in B$.

On the first hand, the above construction provides deformation quantizations of $\mathscr{C}^{\infty}(G) \otimes \operatorname{Pol}\left(\mathscr{G}^{*}\right) \subset \mathscr{C}^{\infty}\left(T^{*} G\right)$, where $\mathscr{G}=\operatorname{lie}(G)$, as explained in [Bieliavsky et al. 2005a]. For $G=\mathbb{R}^{n}$ it reproduces the Moyal star product. On the other hand we have seen that, for $u, v \in \mathscr{C}^{\infty}\left(\mathbb{R}^{2}\right), u \star_{v} v=T_{v}^{-1}\left(T_{v} u \star_{\nu}^{0} T_{v} v\right)$ where $\star_{v}^{0}$ denotes the Moyal star product on $\mathscr{C}^{\infty}\left(\mathbb{R}^{2}\right)$. Restricting to $\mathscr{C}^{\infty}(\mathbb{R}) \otimes \operatorname{Pol}(\mathbb{R}) \subset \mathscr{C}^{\infty}\left(\mathbb{R}^{2}\right)$, the formal equivalence $T_{v}$ is viewed as $\mathrm{i} d \otimes S_{v}$.

Proposition 5.1. The formal star product $\star_{\nu}$ given by (7) coincides with the product underlying the L-R-smash product $\mathscr{C}^{\infty}(\mathbb{R}) \llbracket v \rrbracket \boxminus \operatorname{Pol}(\mathbb{R})$ with $x \rightarrow f=f<x=$ $\frac{1}{2} S_{v}^{-1}\left(\frac{d}{d x}\left(S_{v} f\right)\right)$ and we consider $\operatorname{Pol}(\mathbb{R})$ endowed with its the usual Hopf structure.

Proof. See [Bieliavsky et al. 2005a]: the main fact is that $\mathscr{C}^{\infty}(\mathbb{R}) \llbracket v \rrbracket \curvearrowleft \operatorname{Pol}(\mathbb{R})$ carries the Moyal product for $x \rightarrow f=f<x=\frac{1}{2} \frac{d}{d x}(f)$.

F. Panaite and F. Van Oystaeyen [2005] have shown that if $B$ be a cocommutative Hopf algebra and $C$ a $B$-module algebra, the map $\Phi: C \bigsqcup B \longrightarrow C \sharp B$ with $\Phi(f \llbracket a)=$ $\sum_{(a)} f<a_{(1)} \sharp a_{(2)}$ is an isomorphism of algebras, the multiplication on the smash product $C \sharp B$ being given by $(f \otimes a) \star(g \otimes b)=\sum_{(a)} f\left(a_{(1)} \bullet g\right) \otimes a_{(2)} b$ with $a \bullet g=a_{(1)} \rightarrow g<J\left(a_{(2)}\right), J$ the antipode of $B$. One therefore has

Proposition 5.2. The star product $\star_{v}$ can also be seen as a classical smash product in the sense of Sweedler [1968].

At last, it is shown in [Bieliavsky et al. 2005a] that, under conditions, if $C$ is a Hopf algebra then so is $C \sqcup B$. Seing $\mathscr{C}^{\infty}(\mathbb{R}) \llbracket v \rrbracket$ as $\mathscr{C}^{\infty}(\mathbb{R}) \otimes \mathbb{R} \llbracket v \rrbracket$ and considering 
the natural Hopf structure on the tensor product of the Hopf algebras $\mathscr{C}^{\infty}(\mathbb{R})$ and $\mathbb{R} \llbracket v \rrbracket$ we show in [Bieliavsky et al. 2005a] that the needed conditions are fullfilled. Hence:

Theorem 5.3. There exists a coproduct $\Delta_{v}$, a counit $\epsilon_{v}$ and an antipode $J_{v}$, compatible with $\star_{v}$, such that $\left(\mathscr{C}^{\infty}(\mathbb{R}) \llbracket v \rrbracket \llbracket \operatorname{Pol}(\mathbb{R}), \star_{v}, \Delta_{v}, \epsilon_{v}, J_{v}\right)$ is a Hopf algebra.

Replacing $\mathbb{R}$ by $\mathbb{R}^{n}$ these structural results still hold on the asymptotic expansion of the product described in formula (4).

\section{Acknowledgment}

We thank the referee for suggesting improvements to the original manuscript.

\section{References}

[Bayen et al. 1978a] F. Bayen, M. Flato, C. Fronsdal, A. Lichnerowicz, and D. Sternheimer, "Deformation theory and quantization, I: Deformations of symplectic structures", Ann. Physics 111:1 (1978), 61-110. MR 58 \#14737a Zbl 0377.53024

[Bayen et al. 1978b] F. Bayen, M. Flato, C. Fronsdal, A. Lichnerowicz, and D. Sternheimer, "Deformation theory and quantization, II: Physics applications", Ann. Physics 111:1 (1978), 111-151. MR 58 \#14737b

[Bieliavsky 1995] P. Bieliavsky, Espaces symétriques symplectiques, Ph.D. thesis, Université Libre de Bruxelles, Brussels, 1995.

[Bieliavsky 1998] P. Bieliavsky, "Four-dimensional simply connected symplectic symmetric spaces", Geom. Dedicata 69:3 (1998), 291-316. MR 99c:53055 Zbl 0898.53041

[Bieliavsky 2002] P. Bieliavsky, "Strict quantization of solvable symmetric spaces", J. Symplectic Geom. 1:2 (2002), 269-320. MR 2004c:53150 Zbl 1032.53080

[Bieliavsky et al. 1995] P. Bieliavsky, M. Cahen, and S. Gutt, "Symmetric symplectic manifolds and deformation quantization", pp. 63-73 in Modern group theoretical methods in physics (Paris, 1995), edited by J. Bertrand et al., Math. Phys. Stud. 18, Kluwer Acad. Publ., Dordrecht, 1995. MR 96i:58060 Zbl 0909.53019

[Bieliavsky et al. 2003] P. Bieliavsky, S. Gutt, M. Bordemann, and S. Waldmann, "Traces for star products on the dual of a Lie algebra", Rev. Math. Phys. 15:5 (2003), 425-445. MR 2004e:53119

[Bieliavsky et al. 2005a] P. Bieliavsky, P. Bonneau, and Y. Maeda, "Universal deformation formulae for three-dimensional solvable Lie groups", pp. 127-141 in Quantum field theory and noncommutative geometry (Tohoku, 2002), Lecture Notes in Phys. 662, Springer, Berlin, 2005. MR 2006k:53163 Zbl 1072.53033

[Bieliavsky et al. 2005b] P. Bieliavsky, X. Tang, and Y. Yao, "Rankin-Cohen brackets and quantization of foliation, I: formal quantization”, Preprint, 2005. math.QA/0506506

[Bonneau and Sternheimer 2005] P. Bonneau and D. Sternheimer, "Topological Hopf algebras, quantum groups and deformation quantization", pp. 55-70 in Hopf algebras in noncommutative geometry and physics (Brussels, 2002), edited by S. Caenepeel and F. Van Oystaeyen, Lecture Notes in Pure and Appl. Math. 239, Dekker, New York, 2005. MR 2006d:16062 Zbl 1080.16037

[Bourbaki 1967] N. Bourbaki, Éléments de mathématique, Fasc. XXXIII: Variétés différentielles et analytiques. Fascicule de résultats (Paragraphes 1 à 7), Actualités Scientifiques et Industrielles 1333, Hermann, Paris, 1967. MR 36 \#2161 
[di Bruno 1857] F. di Bruno, "Note sur un nouvelle formule de calcul différentiel", Quart. J. Math. 1 (1857), 359-360.

[Giaquinto and Zhang 1998] A. Giaquinto and J. J. Zhang, "Bialgebra actions, twists, and universal deformation formulas", J. Pure Appl. Algebra 128:2 (1998), 133-151. MR 2000a:16072 Zbl 0938.17015

[Kobayashi and Nomizu 1969] S. Kobayashi and K. Nomizu, Foundations of differential geometry, vol. 2, Interscience Tracts in Pure and Applied Mathematics 15/II, Wiley, New York, 1969. MR 38 \#6501

[Lichnerowicz and Medina 1988] A. Lichnerowicz and A. Medina, "On Lie groups with left-invariant symplectic or Kählerian structures”, Lett. Math. Phys. 16:3 (1988), 225-235. MR 90j:53067 Zbl 0665.53046

[Panaite and Van Oystaeyen 2005] F. Panaite and F. Van Oystaeyen, "L-R-smash product for (quasi) Hopf algebras", Preprint, 2005. math.QA/0504386

[Rieffel 1993] M. A. Rieffel, Deformation quantization for actions of $\mathbf{R}^{d}$, Mem. Amer. Math. Soc. 506, Amer. Math. Soc., Providence, RI, 1993. MR 94d:46072

[Sweedler 1968] M. E. Sweedler, "Cohomology of algebras over Hopf algebras", Trans. Amer. Math. Soc. 133 (1968), 205-239. MR 37 \#283 Zbl 0164.03704

[Trèves 1967] F. Trèves, Topological vector spaces, distributions and kernels, Academic Press, New York, 1967. MR 37 \#726 Zbl 0171.10402

Received March 25, 2005.

PIERRE BIELIAVSKY

DÉPARTEMENT DE Mathématique

UNIVERSitÉ CATHOLIQUE DE LOUVAIN

CHEMIN DU CYCLOTRON, 2

1348 LOUVAIN-LA-NEUVE

BELGIUM

bieliavsky@math.ucl.ac.be

PHILIPPE BONNEAU

Laboratoire de Mathématique et Applications de Metz

UMR CNRS 7122, UNIVERSITÉ DE METZ

ILE DU SAULCY

F-57045 METZ CEDEX 1

FRANCE

bonneau@univ-metz.fr

YOSHIAKI MAEDA

DEPARTMENT OF MATHEMATICS

FACULTY OF SCIENCE AND TECHNOLOGY

KEIO UNIVERSITY

3-14-1, HiYoshi, KонокU-KU

YОКОНАМА, 223-8522

JAPAN

maeda@math.keio.ac.jp 